\title{
INVESTIGAÇÃO DAS ALTERAÇÕES OSTEO-METABÓLICAS E CARDIO-RESPIRATÓRIAS OCORRIDAS APÓS O TREINAMENTO DE MARCHA SOB ESTIMULAÇÃO ELÉTRICA NEUROMUSCULAR EM PACIENTES TETRAPLÉGICOS
}

\author{
INVESTIGATION OF OSTEOMETABOLIC AND CARDIO-RESPIRATORY CHANGES OCCURRING AFTER GAIT \\ TRAINING UNDER NEUROMUSCULAR ELECTRIC STIMULATION IN QUADRIPLEGIC PATIENTS
}

\author{
Daniela Cristina Leite de Carvalho ${ }^{1}$, Alberto Cliquet JR ${ }^{2}$
}

\section{RESUMO}

Este trabalho objetivou avaliar o efeito do treinamento de marcha, com estimulação elétrica neuromuscular (EENM), nos sistemas esquelético e cardio-respiratório de tetraplégicos (C4-C8) completos. Consumo de oxigênio (VO2), produção de dióxido de carbono (VCO2), ventilação minuto $(V E)$, freqüência cardíaca (FC), pressão arterial (PA), gasto energético, análise de marcadores ósseos (osteocalcina, fosfatase alcalina óssea, piridinolina e deoxipiridinolina) e densitometria óssea (DEXA) do colo femoral e fêmur total foram realizados no início e após seis meses. Onze pacientes realizaram marcha sobre esteira ergométrica, com EENM e descarga entre $60-70 \%$ do peso corporal, durante seis meses, duas vezes semanais, vinte minutos diários. Dez pacientes não realizaram marcha. No grupo de marcha, $81,8 \%$ apresentaram aumentos significativos nos marcadores de formação, dos quais 72,7\% também apresentaram diminuição da reabsorção óssea. No grupo controle, 20\% apresentaram aumento na formação óssea. Os resultados da DEXA foram, em geral, opostos àqueles dos marcadores ósseos. Os testes cardio-respiratórios mostraram um aumento significativo para VO2 I/min (36\%), VCO2 (42,97\%), VE $(30,48 \%)$, PAS mmHg (4,8\%) e gasto energético kcal/min $(37,68 \%)$. No grupo controle, apenas o VO2 I/min aumentou significativamente $(26,29 \%)$. O treinamento de marcha com EENM foi mais eficiente para aumentar a taxa de formação óssea e a capacidade aeróbica dos tetraplégicos.

Descritores: Tetraplegia; Marcha; Estimulação elétrica; Densidade mineral óssea; Consumo de oxigênio; Metabolismo; Respiração.

\section{SUMMARY}

This study was aimed to evaluate the effects of gait training with neuromuscular electric stimulation (NMES) on skeletal and cardio-respiratory systems of full quadriplegic patients (C4-C8). Aerobic power (VO2), carbon dioxide production (VCO2), minute ventilation (VE), heart rate (HR), blood pressure (BP), energy consumption, bone markers analysis (osteocalcin, bone alkaline phosphatase, pyridinoline, and deoxypyridinoline) and bone densitometry (DEXA) of the femoral neck and total femur were performed at baseline and six months later. Eleven patients walked on ergometric wake, with NMES and discharge of $60-70 \%$ of body weight, during six months, twice a week, 20 minutes daily. Ten patients did not perform the gait. Within the gait group, $81.8 \%$ showed significant increases of formation markers, of which $72.7 \%$ also presented with reduced bone resorption. Within the control group, $20 \%$ showed increased bone formation. DEXA results were, in general, opposite to those of bone markers. Cardio-respiratory tests showed a significant increase for VO2 I/min (36\%), VCO2 (42.97\%), VE (30.48\%), SBP mmHg (4.8\%) and energy consumption $\mathrm{kcal} / \mathrm{min}(37.68 \%)$. In the control group, only VO2 I/min showed a significant increase (26.29\%). Gait training with NMES was more efficient in increasing bone formation rates and aerobic power in quadriplegic patients.

Keywords: Quadriplegia; Gait; Electrical stimulation, Bone mineral density; Oxygen consumption; Metabolism; Respiration.

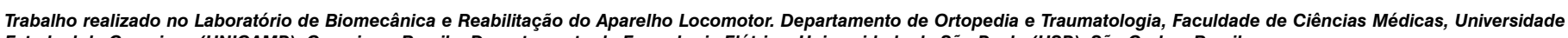
Estadual de Campinas (UNICAMP), Campinas, Brasil e Departamento de Engenharia Elétrica, Universidade de São Paulo (USP), São Carlos, Brasil

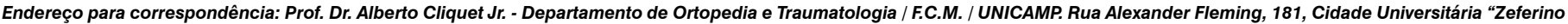
Vaz" s/n - 13083-970 - Campinas, SP / Brasil - E-mails: cliquet@fcm.unicamp.br, dclcarvalho@yahoo.com.br

1 - Fisioterapeuta. Doutora em Cirurgia (Pesquisa Experimental). Pesquisadora Colaboradora Voluntária D.O.T./FCM - UNICAMP.

2 - Professor Titular EESC- USP / FCM - UNICAMP.

Trabalho recebido em: 11/08/05 aprovado em 31/08/05 


\section{INTRODUÇÃO}

Nos últimos tempos, a osteoporose e as doenças cardiovasculares têm aparecido como grandes problemas de saúde pública. Pacientes com lesão medular apresentam uma acentuada perda de massa óssea nos primeiros 3 meses após a lesão, além de apresentarem um maior risco para doenças cardiovasculares que a população normal(1).

Desta forma, programas de reabilitação em pacientes com lesão medular são importantes para melhorar ou manter a saúde dos sistemas esquelético e cardio-respiratório e, conseqüentemente, diminuir os riscos de fraturas nos ossos osteoporóticos e reduzir os riscos de doenças cardiovasculares, também deixando-os aptos a voltarem a andar assim que possível, uma vez que as novas evidências têm mostrado que o treinamento de marcha com estimulação elétrica neuromuscular (EENM) realizado constantemente pode contribuir para a recuperação de movimentos, seja através do padrão gerador de locomoção ou da neuroplasticidade. A possibilidade de recuperação funcional também pode ocorrer através dos avanços médicos (estudos com células tronco)(2) ou tecnológicos (elaboração de estimuladores elétricos que possam ser utilizados para a realização de atividades diárias dos pacientes).

A osteoporose é uma complicação comum associada à lesão medular, devido a paralisia gerar uma diminuição da tensão mecânica sobre os ossos. A ausência de contração muscular e de sustentação de peso aumenta a reabsorção óssea pelos osteoclastos ${ }^{(3)}$, o que promove uma diminuição de massa óssea, tornando os ossos mais frágeis e suscetíveis a fraturas.

A maior perda óssea é observada entre os primeiros quatro a seis meses após o trauma. Após este período a razão de perda óssea diminui e em dezesseis meses a massa óssea se reduz a $2 / 3$ da quantidade original|(3,4).

Com a lesão cervical completa, além da perda significativa da massa óssea, ocorre uma interrupção entre o comando central do sistema simpático com a sua parte periférica(5). A ausência do sistema nervoso simpático altera as respostas cardiovasculares durante o exercício, prejudicando as respostas cronotrópicas e inotrópicas.

Exercício associado à EENM nos membros inferiores recruta grandes grupos musculares, ativa a bomba venosa dos membros inferiores, facilita o retorno venoso, aumenta o volume sistólico, melhora a força de contração do coração e, conseqüentemente, aumenta o fornecimento de $\mathrm{O}_{2}$ aos músculos em atividade ${ }^{(5,6)}$. Além disso, a EENM promove alterações musculares que incluem aumento da resistência muscular, hipertrofia e mudanças histoquímicas (maior número de mioglobinas, mitocôndrias e de densidade capilar), o que aumenta o potencial aeróbico de pacientes com lesão medular. Além disso, a melhora muscular pode beneficiar os ossos, uma vez que um músculo fortalecido gera um maior estresse mecânico sobre o osso que está inserido, aumentando o estímulo a formação óssea(6).

O presente estudo analisou o efeito do treinamento de marcha promovida pela EENM, sobre esteira ergométrica com descarga de $60-70 \%$ do peso corpóreo, durante 6 meses, 2 vezes por semana, sobre a massa óssea e o sistema cardio-respiratório em pacientes tetraplégicos com lesões completas.

\section{MÉTODO}

O estudo clínico foi realizado em pacientes tetraplégicos $(n=21)$, com lesões completas, nível de lesão entre C4 a $\mathrm{C} 8$, que sofreram traumatismo raqui-medular, pertencentes ao Laboratório de Biomecânica e Reabilitação do Aparelho Locomotor, HC/UNICAMP. O estudo foi dividido em 2 grupos: grupo de marcha, formado por 11 pacientes (idade $=33,83$ $\pm 8,73$ anos; tempo de lesão $=78,58 \pm 56,20$ meses; massa corpórea $=64,91 \pm 12,68 \mathrm{~kg}$ e altura $=178 \pm 8,92 \mathrm{~cm})$ e grupo controle, formado por 10 pacientes (idade $=30,9 \pm$ 7,72 anos; tempo de lesão $=51,4 \pm 29,37$ meses; massa corpórea $=65,1 \pm 10,57 \mathrm{~kg}$ e altura $=76,6 \pm 4,81 \mathrm{~cm}$ ).

Os pacientes incluídos no grupo de marcha (GM) realizaram no mínimo 5 meses de exercício de extensão de joelhos através da EENM sobre os músculos quadríceps com o objetivo de aumentar a força e resistência dos músculos quadríceps e, assim, permitir a manutenção da posição ortostática durante os 20 minutos de deambulação.

O treinamento de marcha sobre a esteira ergométrica foi realizado pelo uso combinado de estimulação elétrica de 4 canais, para promover a marcha funcional, e de suspensão dinâmica com descarga entre $60 \%$ a $70 \%$ do peso corporal, para evitar a carga mecânica excessiva sobre os membros inferiores, mas também permitir o toque do calcanhar durante a marcha. O estimulador elétrico utilizado libera uma onda monofásica bipolar, com freqüência de $25 \mathrm{~Hz}$, duração de um trem de pulso de $300 \mu$ s e amplitude de até $200 \mathrm{~V}$ (carga de 1 $k \Omega$ ). O tratamento foi realizado durante 6 meses consecutivos, 2 vezes por semana, com duração de 20 minutos diários, sendo o repouso determinado pelo próprio paciente.

No grupo controle (GC), os pacientes não foram submetidos ao treinamento de marcha induzido pela EENM. Os pacientes que não puderam participar do tratamento proposto, por falta de tempo hábil ou dificuldades de transporte, foram incluídos neste grupo. Os pacientes do GC realizaram apenas fisioterapia convencional 2 vezes por semana (cinesioterapia: manutenção da amplitude de movimento, alongamentos e exercícios ativos na musculatura preservada), sendo que a maioria dos tratamentos também incluiu a manutenção da postura ortostática (através de mesas ortostáticas).

Foram excluídos aqueles pacientes que apresentaram envolvimento do neurônio motor inferior, instabilidade médica e/ou psicológica, outras enfermidades/lesões ou na presença de condição médica ou medicamentosa que interferisse na formação óssea. Este estudo foi aprovado pelo Comitê de Ética em Pesquisa da Faculdade de Ciências Médicas da UNICAMP.

No momento de inclusão na pesquisa e após 6 meses, todos os pacientes foram submetidos a análise de densitometria óssea, coletas dos marcadores bioquímicos do metabolismo ósseo e teste cardio-respiratório.

A densitometria óssea foi realizada na região do colo femoral e fêmur total, utilizando a tecnologia DEXA, modelo DPX-ALPHA, da marca Lunar. A mínima variação significativa para o colo femoral foi de $3,80 \%$ e para o fêmur total de $4,15 \%$. 
Amostras de urina e sangue foram coletadas para as análises dos marcadores ósseos. Foram analisados dois marcadores bioquímicos de reabsorção óssea: piridinolina e deoxipiridinolina livre, os quais foram corrigidos por creatinina urinária. As amostras de urina, protegidas da luz, foram armazenadas a $-20^{\circ} \mathrm{C}$ até o momento da análise. Para a análise foi utilizado o método imunológico baseado em anticorpo contra a piridinolina livre (PYD; Pyrilinks, Metra Biosystems) e deoxipiridinolina livre (DPD; Pyrilinks-D, Metra Biosystems). Também foram analisados dois marcadores bioquímicos de formação óssea: fosfatase alcalina óssea (B-ALP; Alkphase-B, Metra Biosystems) e osteocalcina (OC; Osteocalcin, Metra Biosystems). As amostras de soro foram armazenadas até o momento da análise a $-70^{\circ} \mathrm{C}$. Todas as amostras foram coletadas entre 8:00h e 10:00h da manhã, estando os pacientes em jejum de 12 horas. Para os marcadores do metabolismo ósseo, a mínima variação significativa foi de $21 \%$ para OC, $28 \%$ para B-ALP, $36 \%$ para PYD e $26 \%$ para DPD ${ }^{(7)}$.

O teste cardio-respiratório foi realizado de forma direta e não invasiva, a uma temperatura ambiente de $25^{\circ} \mathrm{C}$, pelo analisador de gases Vmax, modelo 29c da marca SensorMedics. O aparelho foi calibrado antes de iniciar cada teste utilizando gases de referência. Foram analisados os seguintes parâmetros: consumo de oxigênio $\left(\mathrm{VO}_{2}\right)$, ventilação minuto $\left(\mathrm{V}_{\mathrm{E}}\right)$, produção de dióxido de carbono $\left(\mathrm{VCO}_{2}\right)$, freqüência cardíaca $(\mathrm{FC})$ e razão de troca respiratória $\left(\mathrm{R}=\mathrm{VCO}_{2} / \mathrm{VO}_{2}\right)$. Os valores foram registrados a cada 30 segundos e as médias foram obtidas dos últimos 5 valores registrados para cada fase.

Com os valores obtidos de $\mathrm{VO}_{2}$ e $\mathrm{R}$, foi possível analisar a energia (em Kcal) despendida por minuto durante o repouso e o exercício. Através do valor de R obtém-se o equivalente calórico ( $\mathrm{kcal}$ de energia por litro de oxigênio consumido). $\mathrm{O}$ gasto energético foi obtido através da multiplicação do valor médio do $\mathrm{VO}_{2} \mathrm{l} / \mathrm{min}$ pelo valor médio de $\mathrm{Kcal}$ por litro de $\mathrm{O}_{2}$ consumido $\left(\mathrm{Kcal} / / \mathrm{O}_{2}\right)$ obtido do $\mathrm{R}$, pela equação: $\mathrm{R}=\mathrm{Kcal} /$ $\mathrm{IO}_{2}$ e Gasto energético $(\mathrm{Kcal} / \mathrm{min})=\mathrm{VO}_{2} \mathrm{l} / \mathrm{min} \times \mathrm{Kcal} / \mathrm{IO}_{2}$. Todos os pacientes repetiram 3 vezes o protocolo do teste cardio-respiratório, antes do teste oficial, para se acostumarem com o equipamento e com isso permitir a obtenção de dados fidedignos. A freqüência cardíaca foi medida durante o teste, nos intervalos entre sucessivas ondas $R$ do eletrocardiograma da marca SensorMedics.

No GM, o teste foi realizado durante a marcha induzida por EENM sobre esteira ergométrica, auxiliada pela suspensão dinâmica, antes e após o término do tratamento. O teste consistiu de 8 minutos de repouso, 10 minutos de marcha com EENM e 10 minutos de recuperação. As medidas da pressão arterial foram obtidas nas fases de repouso (na posição sentada), marcha (após 10 minutos de marcha) e de recuperação (logo que tenha retomado a posição sentada). Todos os pacientes realizaram o teste inicial a uma velocidade de $0,5 \mathrm{~km} / \mathrm{h}$. O teste final foi realizado na máxima velocidade alcançada por cada indivíduo, variando entre 1,0 a 1,3 km/h.

No GC, o teste consistiu de $8 \mathrm{mi}-$ nutos de repouso, 10 minutos de exercício bilateral de extensão de joelhos na posição sentada utilizando um estimulador de 2 canais e 10 minutos de recuperação.

O GM realizou um treinamento de marcha ao longo de 6 meses, enquanto o GC apenas foi submetido à estimulação elétrica no momento do teste cardio-respiratório, com o objetivo de produzir o efeito agudo da estimulação elétrica nos dois grupos (10 minutos de EENM em ambos os grupos). Além disso, deve ser mencionado que o GC não realizou o protocolo de marcha durante o teste cardio-respiratório
cientes não terem realizado o treinamento prévio de 5 meses de EENM, necessário para capacitá-los a realizar a marcha.

Para avaliar as alterações nos parâmetros metabólicos e cardio-respiratórios $\left(\mathrm{VO}_{2}, \mathrm{VCO}_{2}, \mathrm{~V}_{\mathrm{E}}, \mathrm{FC}\right.$ e $\left.\mathrm{R}\right)$ e os valores de pressão arterial e gasto energético, a Análise de Variância com 2 fatores repetidos foi aplicada para a realização da análise estatística, sendo utilizados níveis de significância de $5 \%(p<0,05)$.

\section{RESULTADOS}

\section{Massa óssea}

A Figura 1 apresenta os resultados dos marcadores ósseos de formação e reabsorção para cada indivíduo do GM. Após 
os 6 meses de treinamento de marcha $(\mathrm{GM})$, resultados mostraram um aumento significativo nos marcadores de formação em $81.8 \%(M=9)$ dos pacientes, sendo que $72,7 \%$ $(\mathrm{M}=8)$ também apresentaram uma diminuição significativa dos marcadores de reabsorção. Além disso, um sujeito apresentou diminuição da taxa de remodelação óssea (indivíduo 10) e um apresentou diminuição da razão de reabsorção óssea (indivíduo 11).

A Figura 2 apresenta os marcadores ósseos de formação e reabsorção óssea para cada indivíduo do GC. Após 6 meses, 30\% dos pacientes não apresentaram alterações significativas nos marcadores ósseos e $20 \%$ apresentaram um aumento significativo nos marcadores de formação óssea. Além disso, 30\% dos pacientes apresentaram diminuição dos marcadores de reabsorção óssea. Um sujeito apresentou aumento da taxa de reabsorção óssea e outro apresentou uma diminuição de marcadores de formação óssea.

A Tabela 1 apresenta os resultados da DMO $\left(\mathrm{g} / \mathrm{cm}^{2}\right)$ no início e após 6 meses no grupo de marcha (GM) e no grupo controle (GC), com as diferenças percentuais entre os valores iniciais e finais.

Os resultados obtidos com os marcadores ósseos, no GM, mostraram que os pacientes $1,6,7,8,10 \mathrm{e}$ 11 não foram correlacionados com os resultados obtidos pela DEXA. Dos pacientes que apresentaram aumento na taxa de formação óssea (9 pacientes), 4 apresentaram aumento na $\mathrm{DMO}$ na região do colo femoral e/ou fêmur total, 5 apresentaram perda da DMO no colo femoral e/ou fêmur total.

No grupo controle, um indivíduo não pôde realizar a DEXA do fêmur porque apresentava uma acentuada contratura flexora do quadril.

Dos 2 pacientes que apresentaram aumento nos marcadores de formação óssea, um paciente apresentou aumento na DMO e o outro apresentou diminuição da DMO. Aqueles que não apresentaram alterações nos marcadores ósseos (3 pacientes), dois pacientes apresentaram diminuição da $\mathrm{DMO}$ e um apresentou manutenção da DMO nas regiões analisadas.
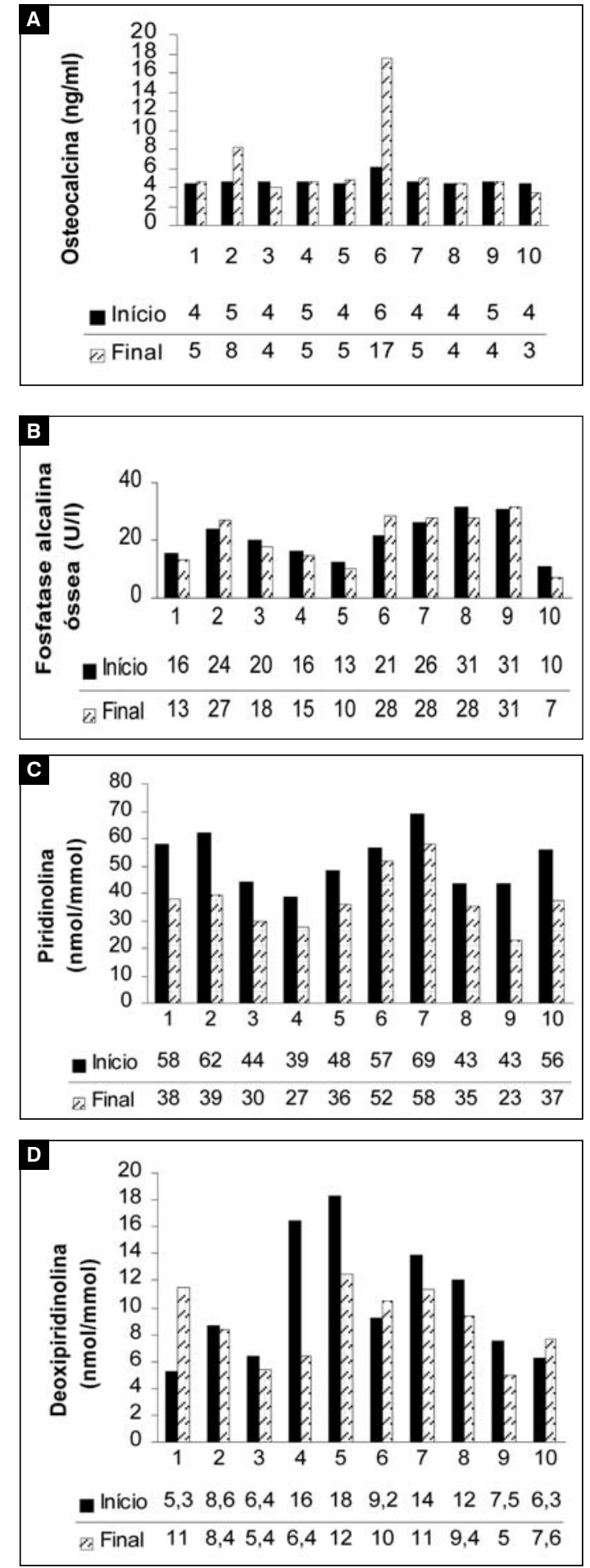

Figura 2 - Resultados dos marcadores ósseos obtidos no início e após 6 meses, no grupo controle. A) Osteocalcina; B) Fosfatase alcalina óssea; C) Piridinolina; D) Deoxipiridinolina.
Teste cardio-respiratório

No teste inicial, no GM os pacientes apresentaram durante a marcha os seguintes valores médios: $\mathrm{VO}_{2}=$ $0,430 \pm 0,09 \mathrm{l} / \mathrm{min}, \mathrm{VO}_{2}=6,96 \pm$ $1,79 \mathrm{ml} / \mathrm{kg} / \mathrm{min}, \mathrm{VCO}_{2}=0,356 \pm$ $0,08 \mathrm{l} / \mathrm{min}, \quad F C=106,23 \pm 17,31$ bpm, $V_{E}=18,93 \pm 2,95 \mathrm{l} / \mathrm{min} \mathrm{e}$ $\mathrm{R}=0.81 \pm 0.06$. Após 6 meses de treinamento, os valores médios obtidos foram: $\mathrm{VO}_{2}=0,586 \pm 0,14$ $\mathrm{l} / \mathrm{min}, \mathrm{VO}_{2}=9,57 \pm 2,54 \mathrm{ml} / \mathrm{kg} /$ $\mathrm{min}, \mathrm{VCO}_{2}=0,509 \pm 0,13 \mathrm{l} / \mathrm{min}$, $\mathrm{FC}=109,23 \pm 15,79 \mathrm{bpm}, \mathrm{V}_{\mathrm{E}}=$ $24,70 \pm 4,62 \mathrm{l} / \mathrm{min}$ e $R=0,85 \pm$ 0,04 . Os resultados mostraram aumentos significativos para $\mathrm{VO}_{2} \mathrm{l} / \mathrm{min}$ (36\%), $\mathrm{VCO}_{2}(42.97 \%), \mathrm{V}_{\mathrm{E}}(30.48 \%)$ e R (4,93\%). A FC não apresentou alteração significativa. Os valores obtidos durante o repouso não sofreram alterações significativas após o treinamento.

Antes do treinamento de marcha ter iniciado, os pacientes apresentaram valores médios de pressão arterial sistólica (PAS) e pressão arterial diastólica (PAD), durante 0 repouso, de 94,16 $\pm 5,15 \mathrm{mmHg}$ e 65,83 $\pm 5,15 \mathrm{mmHg}$ respectivamente. Durante a marcha, a PAS aumentou significativamente para $105 \pm 5,22 \mathrm{mmHg}$, sem alterações na PAD $(70 \pm 7,38 \mathrm{mmHg})$.

Após o período de 6 meses de treinamento, os pacientes apresentaram PAS e PAD, durante o repouso, de 101,67 $\pm 8,35 \mathrm{mmHg}$ e 65,83 \pm 9,0 $\mathrm{mmHg}$ respectivamente, com aumento significativo da PAS. Durante a marcha, a PAS aumentou significativamente para $110,83 \pm$ $6,68 \mathrm{mmHg}(p<0,05)$, sem alteração na PAD $(69,16 \pm 7,92 \mathrm{mmHg})$. A Figura 3 ilustra os valores obtidos antes e após os 6 meses de treinamento para $\mathrm{VO}_{2}, \mathrm{VCO}_{2}, \mathrm{~V}_{\mathrm{E}} \mathrm{FC}$ e PAS durante a fase de marcha. A PAS de repouso também está representada.

No GC, no momento de inclusão na pesquisa, durante o exercício de extensão de joelhos, os pacientes apresentaram os seguintes valores médios: $\mathrm{VO}_{2}=0,232 \pm 0,05 \mathrm{l} / \mathrm{min}, \mathrm{VO}_{2}$ $=3,45 \pm 0,66 \mathrm{ml} / \mathrm{kg} / \mathrm{min}, \mathrm{VCO}_{2}=0,190 \pm 0,04 \mathrm{l} / \mathrm{min}, \mathrm{FC}=$ $67,9 \pm 11,72 \mathrm{bpm}, \mathrm{V}_{\mathrm{E}}=10,96 \pm 1,75 \mathrm{l} / \mathrm{min}$ e $\mathrm{R}=0,82 \pm 0,03$. Após 6 meses, os valores obtidos foram: $\mathrm{VO}_{2}=0,293 \pm 0,09$ $\mathrm{I} / \mathrm{min}, \mathrm{VO}_{2}=4,62 \pm 1,30 \mathrm{ml} / \mathrm{kg} / \mathrm{min}, \mathrm{VCO}_{2}=0,223 \pm 0,07$ 
$1 / \mathrm{min}, \mathrm{FC}=73,32 \pm$ $12,49 \mathrm{bpm}, \mathrm{V}_{\mathrm{E}}=11,65$ $\pm 2,60 \mathrm{l} / \mathrm{min}$ e $\mathrm{R}=0,76$ $\pm 0,07$. Os resultados mostraram um aumento de $26,29 \%$ no $\mathrm{VO}_{2}$ I/min, $17,37 \%$ no VCO ${ }_{2}$, $7,98 \%$ na $\mathrm{FC}, 6,29 \%$ na $V_{E}$, sendo que apenas $\mathrm{O} \mathrm{VO}_{2}$ apresentou aumento significativo $(p<0,05)$ durante $o$ exercício de extensão de joelho. $\mathrm{O} \mathrm{VO}_{2}$ de repouso também aumentou significativamente após 6 meses.

Durante a atividade física, o GM apresentou valores significativamente mais altos (para todos os parâmetros analisados) quando comparado aos valores obtidos no GC (durante o exercício de extensão de joelhos).

Durante a marcha, o gasto energético mé-

\begin{tabular}{|c|c|c|c|c|c|c|c|}
\hline \multirow{2}{*}{$\begin{array}{l}\text { DMO } \\
n\end{array}$} & \multirow[b]{2}{*}{ Grupo } & \multicolumn{3}{|c|}{ Colo femoral } & \multicolumn{3}{|c|}{ Fêmur total } \\
\hline & & Início & Final & $\%$ & Início & Final & $\%$ \\
\hline 1 & GM & 0.923 & 0.863 & $\downarrow 6,5 \%$ & 0.935 & 0.851 & $\downarrow 8,98 \%$ \\
\hline 2 & GM & 0.756 & 0.795 & $\uparrow 5,15 \%$ & 0.686 & 0.682 & $\downarrow 0,58 \%$ \\
\hline 3 & GM & 0.253 & 0.334 & $\uparrow 32,0 \%$ & 0.389 & 0.385 & $\downarrow 1,03 \%$ \\
\hline 4 & GM & 0.674 & 0.673 & $\downarrow 0,15 \%$ & 0.686 & 0.721 & $\uparrow 5,10 \%$ \\
\hline 5 & GM & 0.677 & 0.699 & $\uparrow 3,24 \%$ & 0.638 & 0.654 & $\uparrow 2,5 \%$ \\
\hline 6 & GM & 1.114 & 0.886 & $\downarrow 0,46 \%$ & 0.953 & 0.774 & $\downarrow 8,78 \%$ \\
\hline 7 & GM & 0.977 & 0.904 & $\downarrow 7,47 \%$ & 0.863 & 0.831 & $\downarrow 3,7 \%$ \\
\hline 8 & GM & 0.856 & 0.796 & $\downarrow 7,0 \%$ & 0.801 & 0.760 & $\downarrow 5,12 \%$ \\
\hline 9 & GM & 0.603 & 0.600 & $\downarrow 0,49 \%$ & 0.608 & 0.592 & $\downarrow 2,63 \%$ \\
\hline 10 & GM & 1.079 & 1.022 & $\downarrow 5,28 \%$ & NA & 0.906 & - \\
\hline 11 & GM & 0.838 & 0.692 & $\downarrow 7,42 \%$ & 0.840 & 0.647 & $\downarrow 23,0 \%$ \\
\hline 12 & GC & NA & NA & - & NA & NA & - \\
\hline 13 & GC & 0.759 & 0.692 & $\downarrow 8,82 \%$ & 0.628 & 0.570 & $\downarrow 2,68 \%$ \\
\hline 14 & GC & 0.795 & 0.679 & $\downarrow 14,59 \%$ & 0.763 & 0.627 & $\uparrow 0,4 \%$ \\
\hline 15 & GC & 0.790 & 0.815 & $\uparrow 3,16 \%$ & 0.670 & 0.670 & $\downarrow 0,5 \%$ \\
\hline 16 & GC & 0.647 & 0.669 & $\uparrow 3,4 \%$ & 0.543 & 0.537 & $\downarrow 0,76 \%$ \\
\hline 17 & GC & 0.422 & 0.523 & $\uparrow 23,93 \%$ & 0.557 & 0.513 & $\uparrow 2,17 \%$ \\
\hline 18 & GC & 0.573 & 0.568 & $\downarrow 0,87 \%$ & 1.156 & 1.175 & $\downarrow 0,89 \%$ \\
\hline 19 & GC & 1.115 & 1.068 & $\downarrow 4,21 \%$ & 0.973 & 0.961 & $\downarrow 0,98 \%$ \\
\hline 20 & GC & 0.641 & 0.644 & $\downarrow 0,46 \%$ & 0.717 & 0.717 & $\downarrow 2,02 \%$ \\
\hline 21 & GC & 0.603 & 0.654 & $\uparrow 8,45 \%$ & 0.603 & 0.586 & $\downarrow 1,09 \%$ \\
\hline
\end{tabular}

$\mathrm{NA}=$ não avaliada

Tabela 1 - Valores da DMO $\left(\mathrm{g} / \mathrm{cm}^{2}\right)$ obtidos nas regiões do colo femoral e fêmur total, no início e após 6 meses no grupo de marcha (GM) e no grupo controle (GC). Também estão apresentadas as diferenças percentuais entre os valores iniciais e finais. do, durante a marcha, o gasto energético foi 2,4 vezes maior que o gasto energético de repouso. Após os 6 meses, com a marcha realizada na máxima velocidade alcançada, o gasto energético foi 3,8 vezes maior que o de repouso.

No GC, durante o exercício no teste inicial, o gasto energético foi de 1,12 $\pm 0,25$ e após 6 meses aumentou para 1,39 $\pm 0,46$ (não significativo: $p=0,06$ ). No início do estudo, durante o exercício de extensão de joelhos, o gasto energético foi 1,4 vez maior que o gasto energético de repouso $(0,76 \pm 0,27)$. Após os 6 meses, o gasto energético se manteve em 1,4 vez dio obtido antes do treinamento foi de $2,07 \pm 0,48 \mathrm{kcal} /$ | maior que o de repouso $(0,95 \pm 0,32)$. min e após o treinamento aumentou significativamente para $2,85 \pm 0,71 \mathrm{kcal} / \mathrm{min}(p=0,003)$. No início do estu-

A Figura 4 ilustra o gasto energético $(\mathrm{kcal} / \mathrm{min})$ no momento de inclusão na pesquisa e após 6 meses obtido no GM
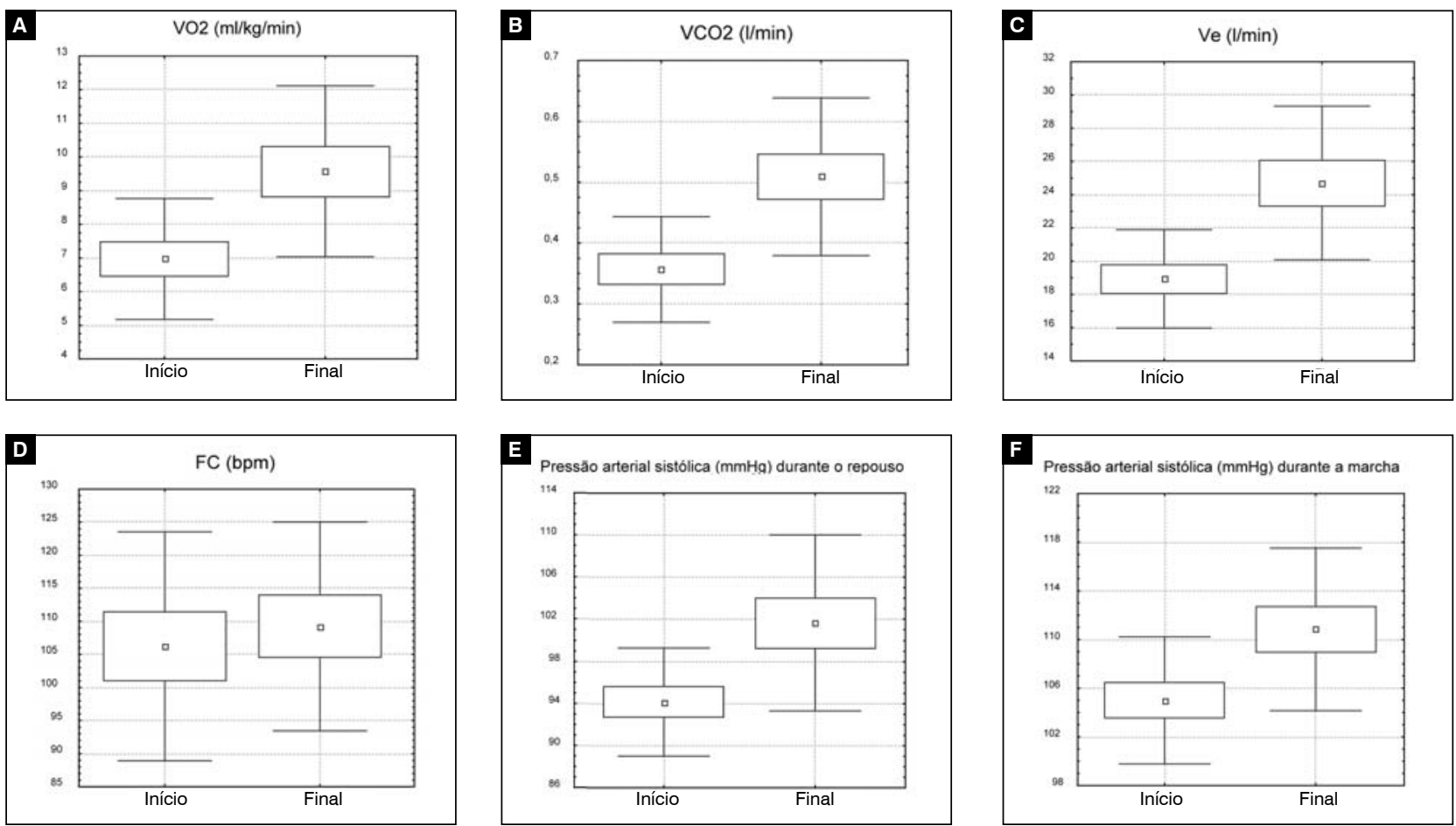

Figura 3 - Valores obtidos no teste cardio-respiratório antes e após os 6 meses de treinamento de marcha. Valores expressos em média \pm DP. A) $\left.\left.\left.\mathrm{VO}_{2}(\mathrm{ml} / \mathrm{kg} / \mathrm{min}), B\right) V_{2} \mathrm{CO}_{2}(I / \mathrm{min}), \mathrm{C}\right) V_{E}(\mathrm{l} / \mathrm{min}), \mathrm{D}\right) \mathrm{FC}(\mathrm{bpm})$, E) PAS ( $\left.\mathrm{mmHg}\right)$ em repouso, F) PAS (mmHg) durante a marcha. 
(durante a marcha) e GC (durante o exercício de extensão de joelhos).

\section{DISCUSSÃO}

Os resultados com os marcadores ósseos mostraram que o treinamento de marcha foi eficaz para aumentar a taxa de formação de osso em $81,8 \%$ dos pacientes incluídos no GM. A análise dos marcadores ósseos é útil para avaliar o comportamento atual do metabolismo ósseo, permitindo avaliar o efeito de terapias sobre a massa óssea em um curto período de tempo ${ }^{(7)}$. A densitometria óssea quando utilizada para monitorar tratamentos é aconselhada que seja realizada com um intervalo de tempo de 1 a 2 anos entre o exame inicial e o final, para aumentar a confiabilidade do resultado ${ }^{(7)}$.

A monitoração óssea em lesados medulares, através da técnica DEXA, deve ser feita com grande cautela, pois associado ao erro de precisão do equipamento, os pacientes podem apresentar espasticidade durante os exames (o que desloca os marcadores de referência das regiões ósseas de interesse), além de ser difícil reproduzir a mesma posição dos membros inferiores ${ }^{(8)}$. O posicionamento alterado dos marcadores pode acarretar diferenças percentuais significativas nos resultados obtidos, principalmente da região proximal do fêmur(8).

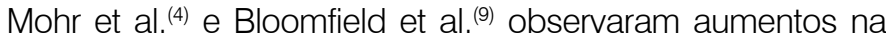
DMO após um treinamento de bicicleta ergométrica associada à EENM (entre 10\% a 30\% na DMO após 6 ou 12 meses de treinamento), os quais foram muito superiores aos resultados obtidos em mulheres pós-menopáusicas em conseqüência aos tratamentos medicamentosos. Aumentos entre 1 a 3\% ao ano são esperados com o tratamento de reposição hormonal e alendronato ${ }^{(10)}$. Embora a falta de carga mecânica pareça ser muito mais prejudicial ao esqueleto (diminuição de cerca de 22\% nos primeiros 3 meses após a lesão) que a falta de hormônio ovariano (diminuição anual de 4 a 5\% da DMO), ainda assim deve ser questionado se exercícios que utilizam a EENM e, conseqüentemente, geram um estímulo mecânico sobre os ossos abaixo do nível de lesão, são eficazes para promover ganhos ósseos tão acentuados em um período curto de tempo.

Além disso, outro aspecto que sugere a imprecisão da técnica DEXA é o fato de muitos pacientes do grupo de marcha terem perdido quantidades significativas de $\mathrm{DMO}$, sendo que todos os pacientes tinham tempo de lesão superior a 24 meses e, baseado na literatura, a maior perda óssea ocorre nos primeiros 3 a 4 meses após a lesão (22-27\% de diminuição óssea), alcançando um novo regime permanente após 16 meses de lesão (perda óssea de cerca de 40\%). Garland et al. ${ }^{(3)}$ não ob- servaram nenhuma diferença da DMO entre 16 meses a 10 anos de lesão.

A eficácia de programas de treinamento aeróbico é freqüentemente determinada por medidas do consumo de oxigênio ${ }^{(11)}$, sendo que as atividades como bicicleta ergométrica e ciclo ergômetro de braço têm se mostrado eficazes no aumento da potência aeróbica. Entretanto, os exercícios com ciclo ergômetro de braço promovem um menor aumento na potência aeróbica, devido ao aprisionamento de sangue acentuado nos membros inferiores, conseqüente à ausência ou diminuição do tônus simpático venoso(5).

Os resultados metabólicos e cardiorespiratórios demonstraram que após 6 meses de treinamento de marcha ocorreu aumento significativo do consumo de oxigênio, com aumento médio do $\mathrm{VO}_{2}$ de $36,28 \%$ (de $0,430 \pm 0,09 \mathrm{l} / \mathrm{min}$ para 0,586 $\pm 0,14 \mathrm{l} / \mathrm{min}$ ), corroborando com os estudos de Mohr et al. ${ }^{(12)} \mathrm{e}$ Goss et al. ${ }^{(11)}$ que também observaram aumentos após o treinamento proposto, embora estes tenham sido em menores proporções (aumento de 18\% e 28\% respectivamente).

O aumento do consumo de oxigênio observado neste estudo ocorreu provavelmente devido às adaptações periféricas, embora possa ter tido alguma influência da melhora do sistema um efeito mais rápido e ocorre devido ao aumento da força e resistência muscular, o que permite ao músculo extrair maior quantidade de oxigênio(5). Para a adaptação central [relacionada com freqüência cardíaca (FC) e com o volume sistólico (VS)] ocorrer é necessário que a atividade física realizada estresse o sistema cardiovascular. A EENM nos membros inferiores ao recrutar grandes grupos musculares, ativa a bomba venosa dos membros inferiores, facilita o retorno venoso, aumenta o débito cardíaco, aumentando a capacidade contrátil do coração pelo mecanismo de Frank-Starling ${ }^{(5,13)}$.

Após os 6 meses de treinamento, a FC dos pacientes tetraplégicos não aumentou significativamente durante o exercício físico devido ao comprometimento do sistema autônomo simpático. O aumento moderado durante a marcha ocorreu, provavelmente, devido à inibição parassimpática e não à ativação simpática.

Desta forma, o provável aumento da capacidade do coração bombear sangue para os tecidos em atividade após o treinamento foi devido ao aumento do retorno venoso (induzido pela EENM), com conseqüente aumento do volume sistólico e da força de contração do miocárdio, sem influência do aumento da FC, uma vez que ocorreu aumento significativo da PAS de repouso (de 94,16 \pm 5,14 para 101,67 \pm 8,35 $\mathrm{mmHg}$ ) e durante a marcha (de $105 \pm 5,22$ para 110,83 
\pm 6,68 mmHg), sem alterações na PAD e na FC. Dela et. al. ${ }^{(13)}$ também observaram durante o exercício em bicicleta ergométrica associada à EENM, em pacientes tetraplégicos, um aumento do débito cardíaco (DC) devido ao aumento do VS, sendo que em pacientes paraplégicos o aumento do DC ocorreu principalmente devido ao aumento da FC. Faghri et al.(6) também observaram um aumento significativo na PAS de repouso, após um programa de treinamento em bicicleta ergométrica associada à EENM (36 sessões, durante 12 semanas), em pacientes lesados medulares crônicos. Eles hipotetizaram que o aumento da PAS após o treinamento possa ter ocorrido devido ao ajuste do sistema hormonal renina-angiotensina do rim, o qual estimula a vasoconstrição das arteríolas, aumentando o volume sanguíneo.

O aumento da PAS, durante a atividade física, reflete o aumento da atividade cardiovascular (aumento da FC e/ou do DC), o que pode ocorrer através do uso da EENM nos membros inferiores. Entretanto, o aumento ocorreu em um grau moderado, porque os pacientes tetraplégicos com lesões completas apresentam uma extensa paralisia muscular associado ao comprometimento do sistema nervoso simpático, prejudicando a resposta cardiovascular durante a marcha.

Os pacientes pertencentes ao grupo controle, também apresentaram melhoras na potência aeróbica (embora em menor proporção), com aumento significativo do $\mathrm{VO}_{2}$ de repouso e durante o exercício de extensão de joelhos.

Segundo Janssen et al. ${ }^{(14)}$ a melhora da capacidade física ocorre após a fase aguda da lesão perdurando até cerca de 48 meses após o traumatismo raqui-medular, devido à fase de adaptação do indivíduo à sua nova condição, o que envolve o aumento da mobilidade através da utilização dos músculos preservados.

Todos os pacientes incluídos no grupo controle realizaram fisioterapia convencional durante o período de 6 meses da pesquisa, sendo que 6 dos 10 pacientes possuíam tempo de lesão inferior a 48 meses, 2 possuíam tempo de lesão de 50 meses e apenas 2 pacientes possuíam tempo de lesão superior a 96 meses. Desta forma, as melhoras metabólicas e cardiovasculares observadas podem ter ocorrido tanto devido à melhora espontânea quanto ao tratamento de fisioterapia convencional que envolveu exercícios da musculatura preservada e o treino de transferências.

Apesar dos pacientes do grupo controle também terem apresentado aumento significativo no $\mathrm{VO}_{2}$ durante o exercício físico, eles apresentaram valores significativamente mais baixos que aqueles alcançados durante a marcha, mesmo na velocidade de $0,5 \mathrm{~km} / \mathrm{h}$.

Após os 6 meses de treinamento, os pacientes do GM apresentaram aumento significativo do gasto energético durante a marcha. Segundo Blair et al. ${ }^{(15)}$, níveis de exercício baixos e moderados, ao aumentar o gasto energético, já são suficientes para diminuir os riscos de doenças cardiovasculares mesmo em pacientes saudáveis. O baixo gasto energético pode ser uma das explicações para o baixo consumo de oxigênio alcançado durante a atividade física.

Desta forma, programas de reabilitação em pacientes com lesão medular são importantes para melhorar ou manter a saúde dos sistemas esquelético e cardio-respiratório, e conseqüentemente, diminuir os riscos de fraturas nos ossos osteoporóticos e reduzir os riscos de doenças cardiovasculares, além de deixá-los aptos a voltar a andar, assim que possível.

\section{CONCLUSÕES}

Os resultados mostraram que o treinamento de marcha foi eficaz para aumentar a taxa de formação óssea (observada através dos marcadores do metabolismo ósseo), além de mostrar que mesmo pacientes tetraplégicos, com lesões completas, podem se beneficiar da atividade física regular, melhorando as respostas metabólicas e cardio-respiratórias.

\section{AGRADECIMENTOS}

Os autores agradecem o suporte financeiro concedido pela FAPESP (\# 2003/05856-9 e \#1996/12198-2).

\section{REFERÊNCIAS BIBLIOGRÁFICAS}

1. Whiteneck GG, Charlifue SW, Frankel HL, Fraser MH, Gardner BP, Gerhart KA, Krishnan KR, Menter RR, Nuseibeh I, Short DJ. Mortality, morbidity, and psychosocial outcomes of persons spinal cord injured more than 20 years ago. Paraplegia. 1992; 30:617-30.

2. de Barros Filho TE, de Oliveira RP, Tsanaclis AM, de Barros EM, Cristante AF, Palma RM et al. An experimental model for the transplantation of fetal central nervous system cells to the injured spinal cord in rats. Rev Hosp Clin Fac Med São Paulo. 2002; 57:256-64.

3. Garland DE, Stewart CA, Adkins RH, Hu SS, Rosen C, Liotta FJ, Weinstein DA Osteoporosis after spinal cord injury. J Orthop Res. 1992; 10:371-8.

4. Mohr T, Podenphant J, Biering-Sorensen F, Galbo H, Thamsborg G, Kjær M. Increased bone mineral density after prolonged electrically induced cycle training of paralyzed limbs in spinal cord injured man. Calcif Tissue Int. 1997; 61:22-5.

5. Figoni SF. Exercise responses and quadriplegia. Med Sci Sports Exerc. 1993; 25: 433-41.

6. Faghri PD, Glaser RM, Figoni SF. Functional electrical stimulation leg cycle ergometer exercise: training effects on cardiorespiratory responses of spinal cord injured subjects at rest and during submaximal exercise. Arch Phys Med Rehabil. 1992; 73:1085-93.

7. Hannon R, Blumsohn A, Naylor K, Eastell R. Response of biochemical markers of bone turnover to hormone replacement therapy: impact of biological variability. J Bone Miner Res. 1998; 13:1124-33.
8. Lekamwasam S, Lenora RS. Effect of leg rotation on hip bone mineral density measurements. J Clin Densitom. 2003; 6:331-6.

9. Bloomfield SA, Mysiw WJ, Jackson RD. Bone mass and endocrine adaptations to training in spinal cord injured individuals. Bone. 1996; 19:61-8.

10. Dawson-Hughes B. Pharmacologic treatment of postmenopausal osteoporosis. In: MJ Favus, editor. An official publication of the American Society for Bone and Mineral Research. Primer on the metabolic bone diseases and disorders of mineral metabolism. 4th ed. Philadelphia: Lippincott-Raven Publishers; 1999. p. 283-8.

11. Goss FL, Mcdermott A, Robertson RJ. Changes in peak oxygen uptake following computerized functional electrical stimulation in the spinal cord injured. Res Q Exerc Sport. 1992; 63:76-9.

12. Mohr T, Andersen JL, Biering-Sorensen F, Galbo H, Bangsbo J, Wagner A, Kjær M. Long-term adaptation to electrically induced cycle training in severe spinal cord injured individuals. Spinal Cord. 1997; 35:1-16.

13. Dela F, Mohr T, Jensen CMR, Haahr HL, Secher NH, Biering-Sorensen F, Kjær. Cardiovascular control during exercise: insights from spinal cord-injured humans. Circulation. 2003; 107:2127-133.

14. Janssen TWJ, Dallmeijer AJ, Veeger D, Van Der Woude LHV. Normative values and determinants of physical capacity in individuals with spinal cord injury. $J$ Rehabil Res Dev. 2002; 39:29-39.

15. Blair SN, Kolh HW, Pattenbarger RS, Clark Jr DG, Cooper KH, Gibbons LW. Physical fitness and all causes of mortality: A prospective study of healthy men and women. J Amer Med Assoc. 1989; 262: 2395-401. 